\title{
Definition and management of odontogenic maxillary sinusitis
}

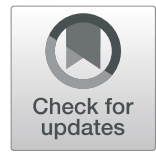

Soung Min $\mathrm{Kim}^{1,2}$ (D)

\begin{abstract}
Background: Maxillary sinusitis of odontogenic origin, also known as maxillary sinusitis of dental origin or odontogenic maxillary sinusitis (OMS), is a common disease in dental, otorhinolaryngologic, allergic, general, and maxillofacial contexts. Despite being a well-known disease entity, many cases are referred to otorhinolaryngologists by both doctors and dentists. Thus, early detection and initial diagnosis often fail to detect its odontogenic origin.

Main body: We searched recent databases including MEDLINE (PubMed), Embase, and the Cochrane Library using keyword combinations of "odontogenic," "odontogenic infection," "dental origin," "tooth origin," "sinusitis," "maxillary sinus," "maxillary sinusitis," "odontogenic maxillary sinusitis," "Caldwell Luc Procedure (CLP)," "rhinosinusitis," "functional endoscopic sinus surgery (FESS)," "modified endoscopy-assisted maxillary sinus surgery (MESS)," and "paranasal sinus." Aside from the PRISMA (Preferred Reporting Items for Systematic reviews and Meta-Analyses) trial, there have been very few randomized controlled trials examining OMS. We summarized the resulting data based on our diverse clinical experiences.

Conclusion: To promote the most efficient and accurate management of OMS, this article summarizes the clinical features of rhinosinusitis compared with OMS and the pathogenesis, microbiology, diagnosis, and results of prompt consolidated management of OMS that prevent anticipated complications. The true origin of odontogenic infections is also reviewed.

Keywords: Rhinosinusitis, Odontogenic maxillary sinusitis (OMS), Functional endoscopic sinus surgery (FESS), Modified endoscopy-assisted maxillary sinus surgery (MESS), Odontogenic infection
\end{abstract}

\section{Background}

Maxillary sinusitis of odontogenic or dental origin, also known as chronic maxillary sinusitis of dental origin, or odontogenic maxillary sinusitis (OMS), is a comparatively well-known disease in dental, otorhinolaryngologic, and allergic contexts. Any diseases arising from dental or dentoalveolar structures could affect the Schneiderian membrane (SM), leading to diverse pathologic disease presentations in the maxillary sinus. Exact and accurate diagnosis of odontogenic origin is necessary to avoid the long-term administration of inappropriate medications or unnecessary surgical management.

\footnotetext{
Correspondence: smin5@snu.ac.kr; smin_kim@hanmail.net

${ }^{1}$ Oral and Maxillofacial Microvascular Reconstruction LAB, Ghana Health Service, Brong Ahafo Regional Hospital, P.O. Box 27, Sunyani, Brong Ahafo, Ghana

${ }^{2}$ Department of Oral and Maxillofacial Surgery, Dental Research Institute, School of Dentistry, Seoul National University, 101 Daehak-ro, Jongno-gu, Seoul 110-768, South Korea
}

The aim of this article is to provide information about the pathophysiology of OMS for comparisons with chronic or acute maxillary sinusitis, including chronic rhinosinusitis (CRS) and acute bacterial rhinosinusitis (ABRS). Clinical features including the pathogenesis and microbiology of OMS are reviewed, and appropriate management with accurate diagnosis, prompt consolidated treatment, and prevention of anticipated complications is summarized.

\section{Main text}

We conducted a search of recent, up-to-date databases including MEDLINE (PubMed), Embase, the Cochrane Library, and other online tools using keyword combinations of "odontogenic," "odontogenic infection," "dental origin," "tooth origin," "sinusitis," "maxillary sinus," "maxillary sinusitis," "odontogenic maxillary sinusitis," "Caldwell Luc procedure (CLP)," "rhinosinusitis," "functional endoscopic sinus surgery (FESS)," "modified endoscopy-assisted 
maxillary sinus surgery (MESS)," and "paranasal sinus." The results are summarized based on our diverse clinical experiences.

A statement of ethics approval was provided by the Department of Oral and Maxillofacial Surgery at Seoul National University Dental Hospital, with the approval of the Institutional Review Board of Seoul National University (S-D20170005).

\section{Chronic and acute rhinosinusitis}

The importance of appropriate diagnosis and management of chronic or acute rhinosinusitis cannot be emphasized enough, because nasal or sinus problems including nasal stuffiness, nasal airway obstruction, nasal drainage, and postnasal drip are very common [1-3].

\section{Classification of rhinosinusitis}

Rhinitis has been confused with rhinosinusitis and described using terminology that is a more accurate definition for describing inflammations of the inner nasal cavity involving the paranasal sinuses. A diagnosis of rhinosinusitis requires two of the following symptoms: nasal obstruction, middle facial pain, mucopurulent discharge, and decreased smell, with additional observation of mucosal inflammation required for final consolidated diagnosis. The treatment of rhinosinusitis varies according to etiology, and initial differentiation between acute and chronic forms should be made while considering the patient's previous history, present symptoms, and the results of nasal endoscopic examination or careful intraoral inspection. Evidence-based therapy may be initially managed by physicians in cases of acute or chronic rhinosinusitis, and more difficult symptoms that are refractory to avoidance can be referred to an allergist for further immunotherapy [4]. Acute rhinosinusitis (ARS) is defined according to symptom duration as follows: infectious ABRS, with purulent nasal discharges, obstruction, and pain with sensation of fullness within 4 weeks; subacute rhinosinusitis (SRS) between 4 and 8 weeks; and CRS with symptoms lasting more than 8 weeks despite treatment with medications. Rhinosinusitis has been also classified into allergic or non-allergic, occupational, and other types of rhinitis syndromes.

\section{Mucociliary clearance functions of SM}

The pseudostratified ciliated columnar epithelium, known as $\mathrm{SM}$, lines the inner respiratory mucosa of the maxillary sinuses. The SM produces mucus that moves to the ostium for drainage into the nasal cavity against normal gravity, with movement of cilia around the maxillary sinus occurring in a synchronized pattern (Fig. 1). This mucus, passing from the nasal cavity to the nasopharynx, is swallowed and passes into the esophagus and stomach. Any interruption of these basic movements of mucus by reduced ciliary activity or obstruction of ostia can result in sinus disease and symptoms. Each ostium of the anterior ethmoidal sinus, frontal sinus, and maxillary sinus is closely approximated in the middle nasal meatus, and together, these comprise the osteo-meatal unit (OMU). Thus, any inflammation or blockage of the OMU will induce sinusitis, including cases involving several sinuses, referred to as pan-sinusitis.

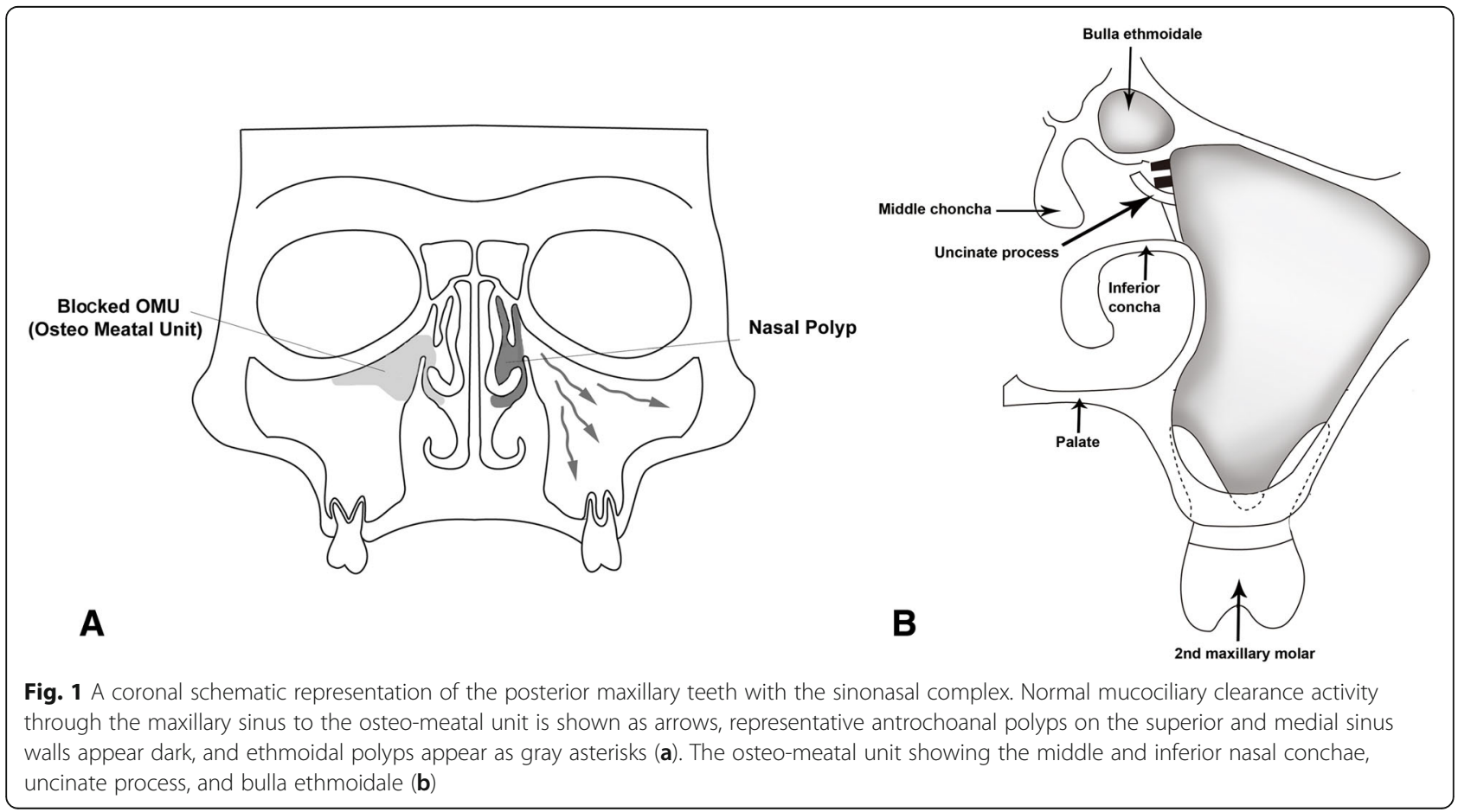


The epithelial cells of SM play essential roles in mucociliary clearance (MCC) and keeping the upper airway clean by driving continuous ciliary beating to move inhaled foreign bodies, bacteria, fungi, and viruses toward the oropharyngeal airway. These basic protective functions are aided by the airway epithelium with mucin secretions that create ion or fluid transport to maintain mucous viscosity. Several chemokines are secreted according to pathogen exposure levels to activate inflammatory or protective immune pathways by recruitment of macrophages, dendritic cells, eosinophils, neutrophils, T cells, and NK cells (Fig. 2) [4, 5]. Several cytokines, including IL-1 $\beta$, IL-6, TNF $\alpha$, IL-8, and monocyte chemotactic protein 1, are also released. These epithelial cells of SM are connected by tight junctions to form a physical defensive wall, and mucociliary transport is managed by the formation of reactive oxygen and nitrogen species through control of antimicrobial peptides such as lactotransferrin, lysozyme, and defensins (Fig. 2).

\section{Definition and course of $A B R S$}

The main etiology of ABRS is infection by native bacteria in ambient air through the upper aerodigestive tract. Thus, the first goal of ABRS management is reducing bacterial infections accompanied by symptomatic improvement. Most cases of inflammatory sinusitis, including ABRS, occur within 7 to 10 days after upper respiratory tract viral infection. ABRS patients can recover from viral infections but may have continuous symptoms such as facial pain and nasal congestion with rhinorrhea. Nasal or sinus stasis may occur because of reduced MCC activity and the high anatomical position of the ostium. Sometimes ethmoidal or antrochoanal polyps, known anatomical variations, aggravate stasis by blocking physiologic drainage through the middle nasal meatus (Fig. 1). Due to the variation in pathophysiologic entities in OMS, exact management of the original dental problems should follow ABRS symptom relief. Amoxicillin can usually be administered to ABRS patients according to individual clinical progress and comorbidities, while computed tomographic scans are helpful for the objective diagnosis of complicated symptoms or other severe complications such as intracranial extension [6].

\section{Definition and course of CRS}

Typical CRS is defined as having more than two of the following symptoms for more than 12 weeks: facial pressure pain, decreased smell, nasal congestion, rhinorrhea, or postnasal drip. Facial pressure pain is usually described as a dull and localizing pressure pain in the upper cheek with continuous headache on the same side of the forehead. Decreased smell can be divided into partial hyposomia and total anosmia, which are both related to anterior ethmoidal mucosal opacifications. Sometimes such patients complain of reduced taste sensation, known as ageusia. Nasal congestion is also expressed as nasal stiffness or fullness and nasal cavity blockage. Anterior or posterior rhinorrhea is defined as a thick yellow or brown mucus discharge, which is more common in ABRS than in CRS patients.

CRS etiology is associated with diverse anatomical variations and inhalation of foreign bodies. Cigarette smoking or allergic rhinitis is also known to influence CRS, along with social economic status. Anatomical variations, such as deviated septum or middle nasal turbinate, and abnormal Haller cell size or agger nasi cells, can cause obstruction of the OMU and consequently induction of CRS. Several known environmental air irritants, such as sulfur dioxide, ozone, and formaldehyde, have

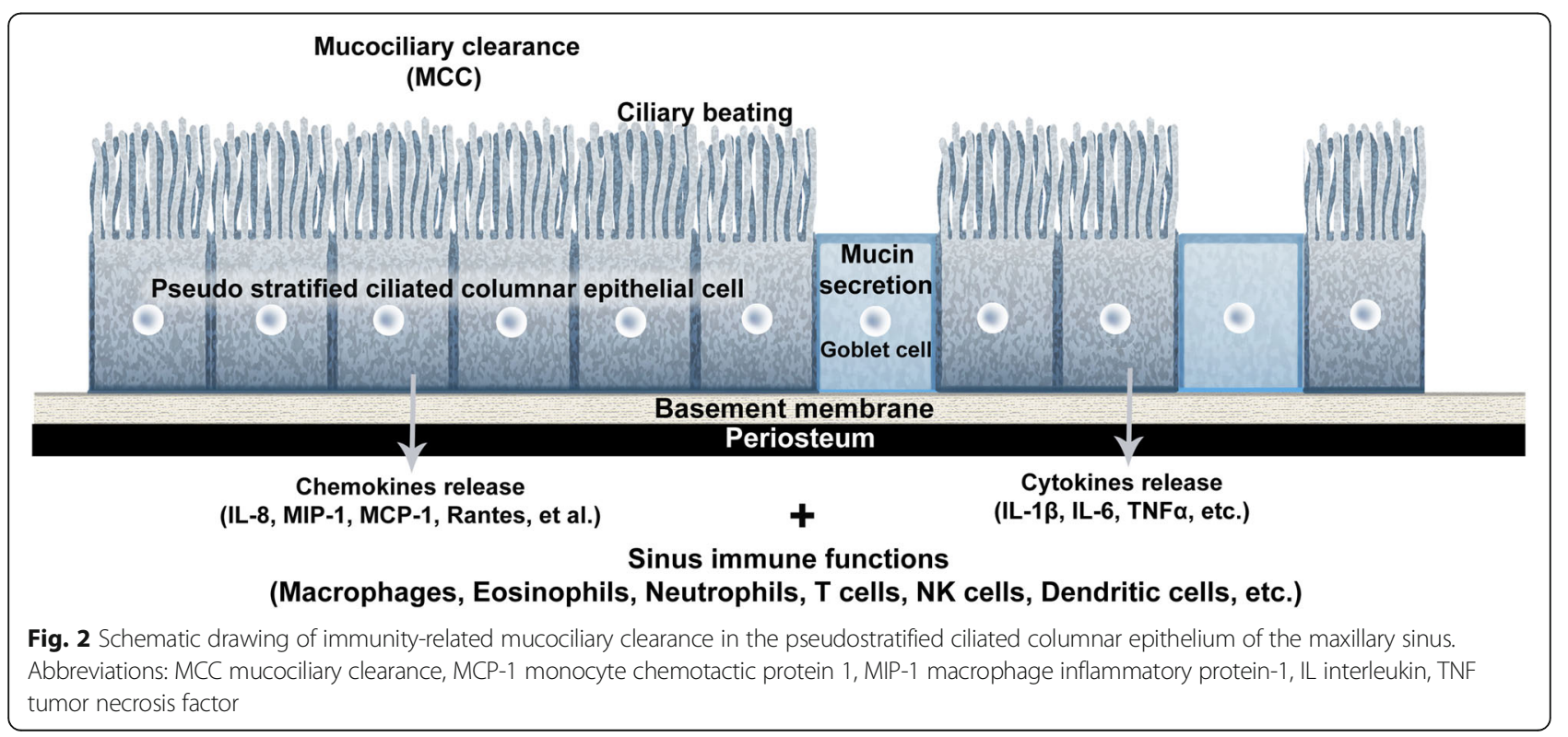


also been shown to impact MCC function. Allergic rhinosinusitis has underlying genetic or immune factors related to the development of CRS $[4,5]$.

CRS is classified as CRS without polyp or allergic fungal sinusitis according to polyposis or fungal infections. More recently, the pathogenesis of CRS has been shown to involve immune responses changes. Polyps or cystic fibrosis results in abnormal changes of the sinonasal epithelium that alter MCC function [5-7]. Inhalation exposure to irritants, such as bacteria including Staphylococcus aureus (S. aureus), fungi, viruses, and proteases, degrades the functions of respiratory epithelial barriers. Dysregulated epithelial cells may release inflammatory molecules such as thymic stromal lymphopoietin, which can aggravate the development of type 2 immune inducer responses in CRS in nasal polyp patients. Innate immune cells such as type II innate lymphoid, mast cells, and eosinophils are increased, and these cells can release type 2 cytokines including IL-4, IL-5, and IL-13 that further perpetuate the ongoing inflammatory response [5-7]. In contrast, adaptive immune cells such as dendritic, $T$ helper type 2 , native $B$, and activated plasma cells are also increased in CRS with nasal polyps, and thus contribute to increased local production of antibodies within the sinonasal tissue [6,7]. Type 2 cytokines are also thought to contribute to decreased tissue plasminogen activator and increased Factor XIIIa levels, which in turn lead to increased fibrin deposition and cross-linking within nasal polyps (Fig. 2).

Treatment of CRS is based upon severity and etiology. Corticosteroids and additional antibiotics can be helpful when coupled with saline irrigation through the nasal cavity. Discrimination between diverse causes of CRS while ruling out other symptoms is essential to ensure good outcomes after CRS management [8]. However, all avenues of clinical management for CRS result in limited outcomes because of the heterogeneous pathology of CRS.

\section{Odontogenic maxillary sinusitis}

The incidence of OMS has likely been underreported, with $10-12 \%$ of OMS cases attributed to odontogenic infections [9-12] in the otorhinolaryngological literature. Studies that are more recent suggest a much higher incidence than previously reported, with chronic maxillary sinusitis (CMS) comprising $30-40 \%$ of all cases of CMS [13].

\section{Development and growth of maxillary sinus}

The maxillary sinus may occasionally be absent or hypoplastic during development and show growth spurts at $0-3$ and 7-12 years, which correspond with the development and eruption of the permanent dentition and pubertal facial growth [14, 15]. A pneumatization process continues with maxillary sinus growth throughout the whole lifetime, until the sinus floor is at a level below the nasal floor after loss of the involved teeth. The first and second molars are close to the inferior wall of the maxillary sinus, with the premolar teeth less so and ectopic canine teeth only occasionally adjacent. The OMU is located superiorly on the medial wall and average $2.4 \mathrm{~mm}$ in diameter, while the bony window is much larger [14-16]. The effective opening of the ostium may be reduced by the projection of the uncinate process, which is an extension of the inferior turbinate and the surrounding soft tissues (Fig. 1).

The bony wall that separates the maxillary sinus from the dental roots varies, ranging from complete loss in which the roots are covered only by SM to a thickness of more than $12 \mathrm{~mm}$. The mean distance between the maxillary molar and premolar roots and the maxillary sinus is $1.97 \mathrm{~mm}$, which suggests that the tips of the roots might project into the floor of the sinus, causing small elevations or prominences along the SM [17, 18]. These intimate anatomical relations of the upper molar teeth to the maxillary sinus facilitate the development of periapical or periodontal odontogenic infection inside the maxillary sinus [19] (Fig. 3).

\section{Definition and etiology of OMS}

A variety of odontogenic diseases involve the maxillary sinus, from the lining of the sinus to the adjacent paranasal sinuses and dental tissues, or from the adjacent bone with expansion into the sinus (Fig. 3). Tooth extraction-related OMS is the most common cause (Fig. 4), alongside other dento-alveolar lesions including dentigerous cysts (Fig. 5), radicular lesions (Fig. 6), dental caries (Fig. 7), impacted teeth, and root infections of external resorbed molars (Fig. 8). The molar region has a frequency of involvement of $47.68 \%$, followed by the first molar $(22.51 \%)$, the third molar (17.21\%), and the second molar (3.97\%). The premolar region is involved in $5.96 \%$, followed by the canine in $0.66 \%$ [20, 21].

Otorhinolaryngologists and medicinists have defined OMS as an iatrogenic dental disease, but these explanations are inaccurate due to deficiencies in knowledge regarding the anatomy and physiology of the maxillary sinus. Oroantral fistula (OAF) with or without tooth extraction, retained root infections, periodontitis, and other related odontogenic pathologies (Fig. 3) are the most common etiologies of OMS. Inferior maxillary sinus floor elevation after bone graft procedures, sinus floor perforation or poor positioning during dental implant fixture installation, extruded endodontic obstructive materials, foreign bodies present after apicoectomy, and surgical extraction of an impacted third molar may all be considered iatrogenic causes of OMS. However, all these procedures can be made safer when clinicians use safe, accurate approaches, even in patients with severely pneumatized maxillary sinuses. 


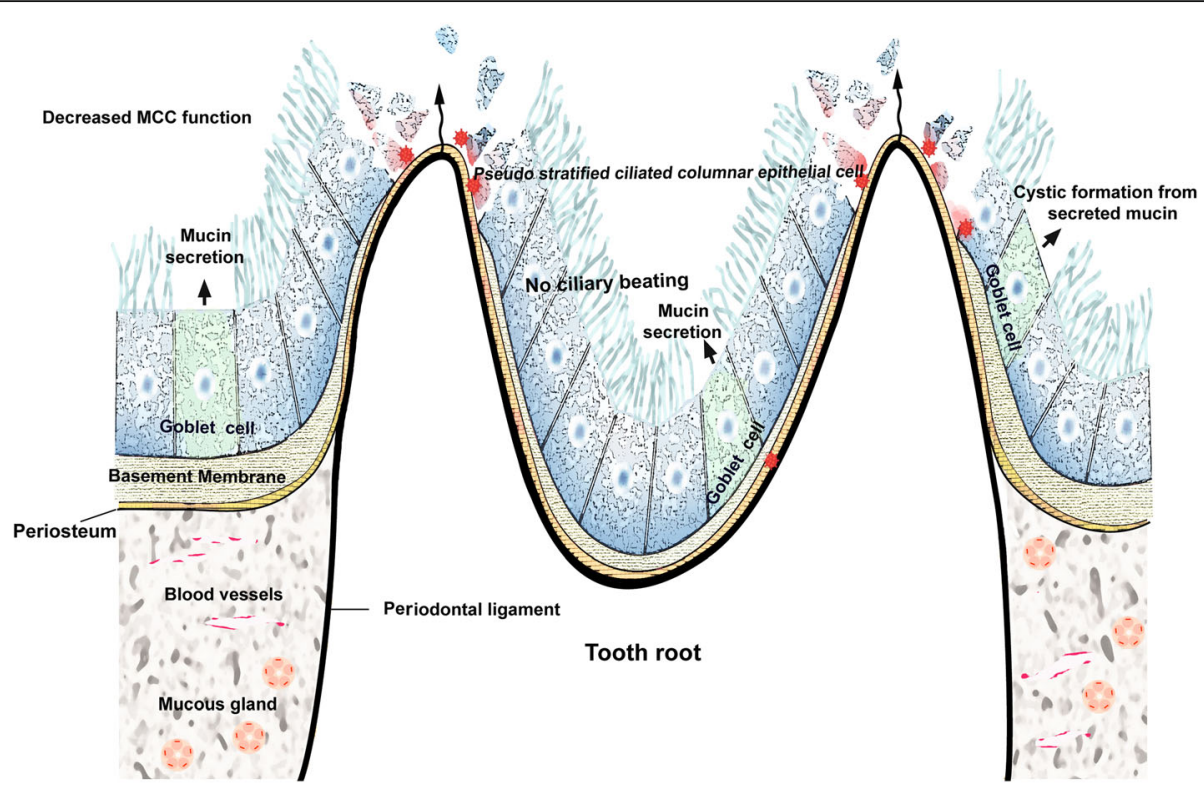

Fig. 3 Schematic drawing of the breakdown of mucociliary clearance (MCC) function due to odontogenic infection. The dysregulated epithelial mucosal barrier is widened according to infection severity and duration

\section{Diagnosis of OMS}

The most frequent clinical features of OMS can be divided into dental and sinonasal symptoms. Dental symptoms including involved tooth pain and hypersensitivity are not easily identified as odontogenic causes, but infrequent dental discomfort may occur after OMU patency preservation with continuous progression of maxillary sinus symptoms [11, 22, 23]. Representative sinonasal symptoms are unilateral cheek pain with nasal obstruction, purulent rhinorrhea, foul odor, foul taste, headaches, anterior maxillary tenderness, and postnasal drip. These symptoms cannot be distinguished from other causes of rhinitis, nor can any typical symptom be considered predominant in OMS [20, 21].

Unilateral nasal obstruction with facial pain and pressure is also a common symptom in OMS, and foul odor with rotten taste combined with tooth pain appears to clinically differentiate CMS and OMS [22, 23]. The most common dental causes are periapical abscess, periodontal disease, post dental extraction, OAF, and undetected foreign bodies in the sinus. OMS can also develop due to maxillary osteomyelitis, radicular cysts, mechanical injury of the sinus mucosa during root canal treatment, overfilling of root canals with endodontic material, incorrectly positioned dental implants, and improperly performed sinus augmentation [24-26].

\section{Pathogenesis of OMS}

Excluding close anatomical relationships, which can be thought of as facilitating inflammatory spread from the maxillary molars and premolars to the inferior maxillary sinus wall, many other conditions can contribute to the pathogenesis of OMS. Endo-antral syndrome was presented as a spreading pulpal disease by Selden [27-29], characterized by pulpal disease, periapical radiolucency or lamina dura loss on radiographs, faintly

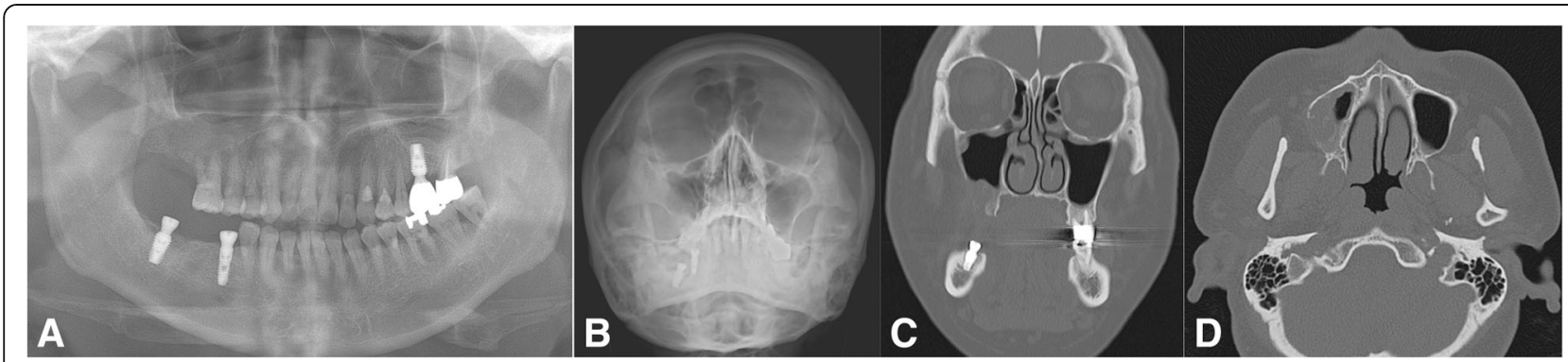

Fig. 4 Chronic oronasal fistula after second molar extraction with several points of alveolar bony resorption indicates odontogenic maxillary sinusitis. Preoperative panoramic (a), Water's (b), coronal CT scan (c), and axial CT scan (d) views 


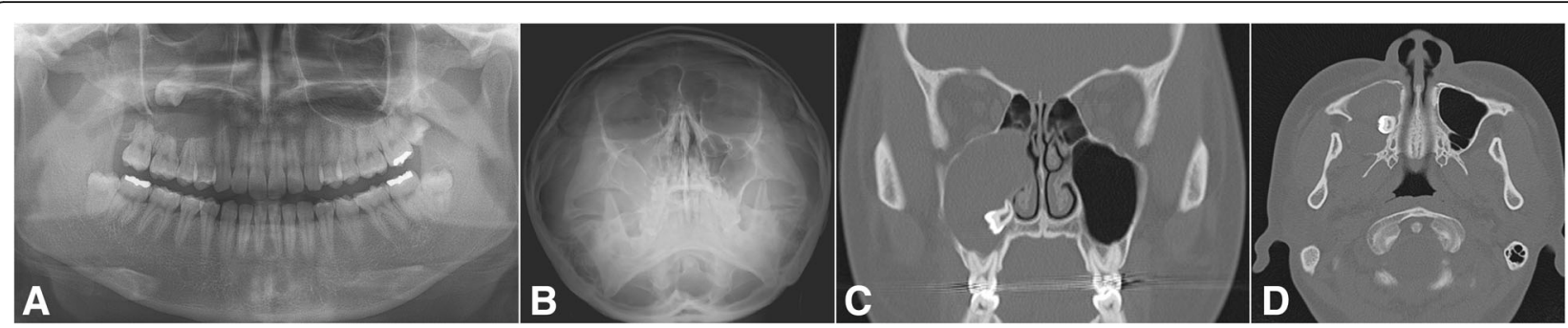

Fig. 5 A case of odontogenic maxillary sinusitis originating from a tooth bearing a huge cyst in the right maxillary sinus. Preoperative panoramic view (a), Water's view (b), coronal CT scan view showing a bony expansible cystic mass with ostium obstruction (c), and axial CT scan view showing the posterior expansional mass (d)

radiopaque mass bulging into the sinus wall, and variable radiopacities on the inferior sinus wall. Rapid spreading of dental infections may also lead to infraorbital cellulitis, transient blindness, and even lifethreatening cavernous sinus thrombosis [23, 27].

The prevalence of OMS with secondary periapical lesions is $16-65 \%$ [28-30], and its management is more complicated than cases with only primary lesions [31]. Endodontic lesions spreading into the sinus are characterized by epithelial cells surrounded by connective inflammatory tissues [32-34]. Endodontic lesions could become evoluted over time during the acute or invasive phase, as well as the chronic phase. The acute phase is much more invasive and can cause the spread of bacteria directly into the sinus cavity and SM, causing hypertrophic reactions. Furthermore, if endodontic treatment does not eliminate the causative microorganisms, these hypertrophic reactions can lead to recurrent periodontitis or secondary periapical lesions [31, 35].

Other causes of OMS are SM mucosal edema with inflammatory cell infiltrates, odontogenic or mucous retention cystic formation, hypertrophic scarring or granulation, hyalinization, and necrotic odontogenic infections [14]. Apical lesions may lead to inflammation and thickening of the SM adjacent to the involved tooth roots and consequently to periapical osteitis with sinus mucosal hyperplasia [36, 37].

\section{Microbiology and biofilm hypotheses}

OMS has basic polymicrobial characteristics, with predominantly anaerobic bacteria in both the oral cavity and upper respiratory tract. Aerobic Staphylococcus aureus and Streptococcus pneumonia (S. pneumonia) and anaerobic Peptostreptococcus and Prevotella spp. are found in more than $75 \%$ of cases, while methicillinresistant Staphylococcus aureus is found in 10-12\% of OMS patients [38, 39]. Intraradicular bacterial and fungal genera and species such as Streptococcus, Propionibacterium, and Candida albicans may cause secondary periapical lesions, and more than 158 bacterial species and 3 fungal species may be also involved in the etiology of secondary periapical infections with the most common being Enterococcus faecalis bacteria [40, 41].

OMS-related periapical lesions have biofilm granules related to granulomatous lesions [42]. The bacterial biofilm (BB) hypothesis of OMS was recently proposed, implicating dynamic polymicrobial communities with slow replicating strains embedded in the extracellular polymeric matrix including exopolysaccharides, proteins, and nucleic acids [38]. These matrix substances are arranged in discrete layers between metabolically active strains in active outer coatings exposed to higher oxygen and nutrient concentrations, with quiescent bacteria in the deeper and inactive anaerobic core [39]. Deeper layers are relatively protected from antibiotics, detergents, and other antimicrobial compounds under humoral or

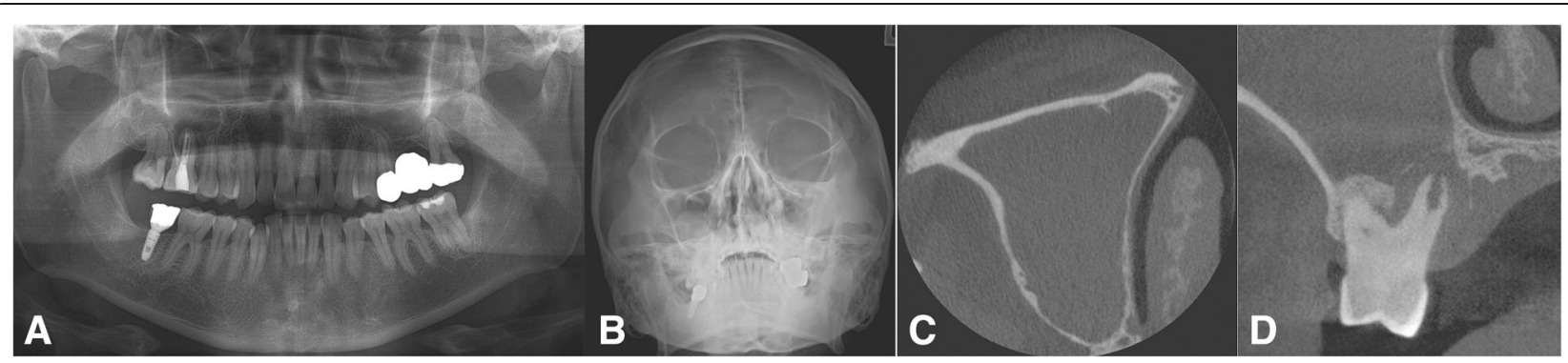

Fig. 6 A representative case of odontogenic maxillary sinusitis originating from an apical lesion in the right upper second molar. Preoperative panoramic (a), Water's (b), axial cone-beam CT scan (c), and coronal cone-beam CT scan (d) views 


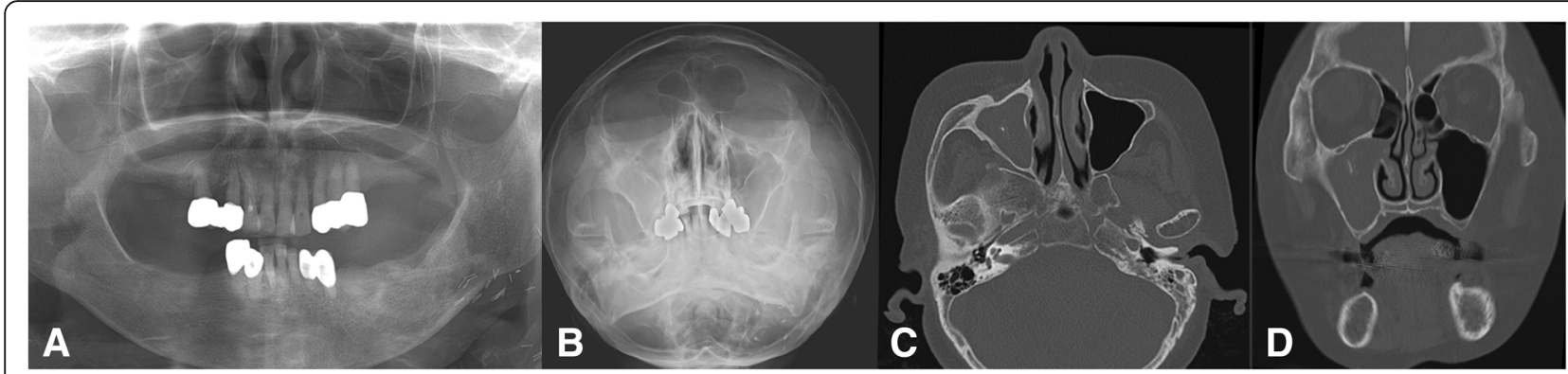

Fig. 7 Chronic maxillary sinusitis originating from the right second premolar, the crown of which was analyzed or continuous denture loading. Preoperative panoramic view (a), Water's view (b), and axial CT scan view showing radiopacity in the middle of the sinusitis suspicious of fungal ball (c), and coronal CT scan view showing definite fungal maxillary sinusitis with ostium obstruction (d)

cellular immunity [39], thus making them responsible for recalcitrant chronic infections.

BB has a detection rate of $70 \%$ in $25-100 \%$ of CRS samples [43-47]. BB may also act as a mechanism in OMS with chronic paranasal sinus inflammation and respiratory mucosal biofilm [48, 49]. The main pathogens involved in OMS $\mathrm{BB}$ are $S$. aureus, Haemophilus influenza ( $H$. influenza), $P$. aeruginosa ( $P$. aeruginosa), coagulase-negative staphylococci, Moraxella catarrhalis, S. pneumoniae, and fungal species [40, 41], as well as anaerobic species. Sometimes, displaced implants or endodontic materials inside the maxillary sinus do not result in signs of maxillary sinusitis, despite the fact that odontogenic infections are the cause of maxillary sinusitis in most OMS cases. Excluding concomitant nasal conditions including OMU status, the presence of $\mathrm{BB}$ should be determined when OMS develops [43, 44, 50, 51].

Actinomyces in the maxillary sinus Actinomyces spp., including $A$. israelii and $A$. radicidentis, can be found on extraradicular granules inside the maxillary sinus due to their peculiar surface structures that allow epithelial attachment to inflammatory cells and oral bacteria $[52,53]$. The extraradicular lesions caused by actinomycosis are resistant to host immune system responses, antibiotics, and orthograde treatment because orthograde endodontic treatment by itself does not reach the extraradicular bacteria [26]. The difficulty of treating Actinomyces- involved maxillary sinus infections indicates that alternative means of treating apical periodontitis, or apical surgery, may be required for the successful management of Actinomyces-related OMS [24]. Despite difficulty in distinguishing apical periodontitis caused by extraradicular or intraradicular microorganisms based on clinical signs and radiography, actinomycosis might be considered to be related to specific clinical signs and symptoms of OMS [25].

Fungal sinusitis In immune-compromised OMS patients, including those with poorly controlled diabetes mellitus, HIV infections, or undergoing chemotherapy, fungal infections are also seen in the maxillary sinus. Aspergillosis or mucomycosis may extend to the orbital wall, temporal fossa, and even to the brain, thus producing signs and symptoms suggestive of malignant disease [54]. Most of these fungal species are inhaled through the respiratory tract and persist in the sinus mucosa by making molds and spores. The foci of infection may lead to dystrophic calcification and the formation of rhinoliths, which may be seen on dental radiographs, with large rhinoliths known as fungal balls. When fungal infection occurs with relation to dental foreign materials, the infection is normally contained within the confines of the maxillary sinus (Fig. 9) [55].

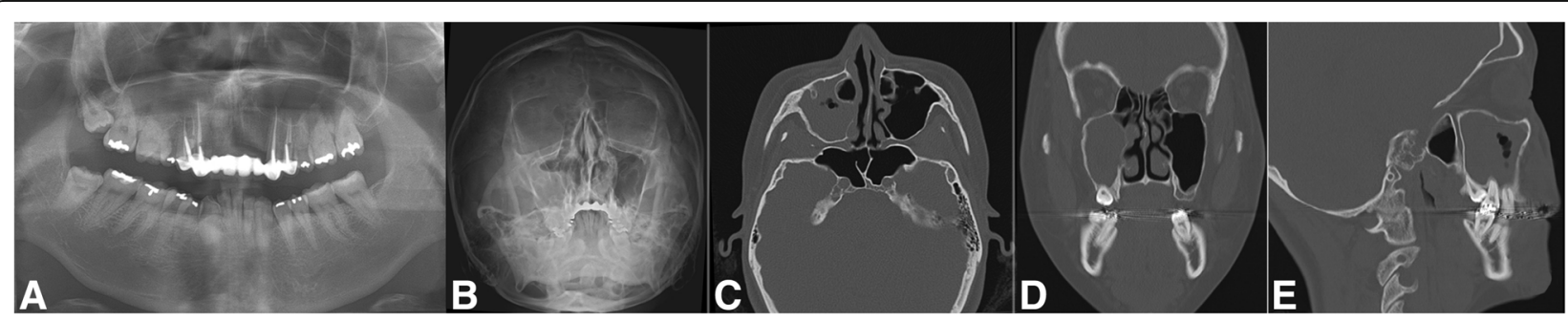

Fig. 8 Odontogenic maxillary sinusitis originating from both an impacted third molar and a root infection of an external resorbed first molar in the right maxillary sinus. Preoperative panoramic view (a), Water's view (b), and axial CT scan view showing an air-bubble and including a sinus mass (c). A coronal CT scan view showing the direct involvement of the right first molar (d) and a sagittal CT scan view showing direct involvement with three molars (e) 


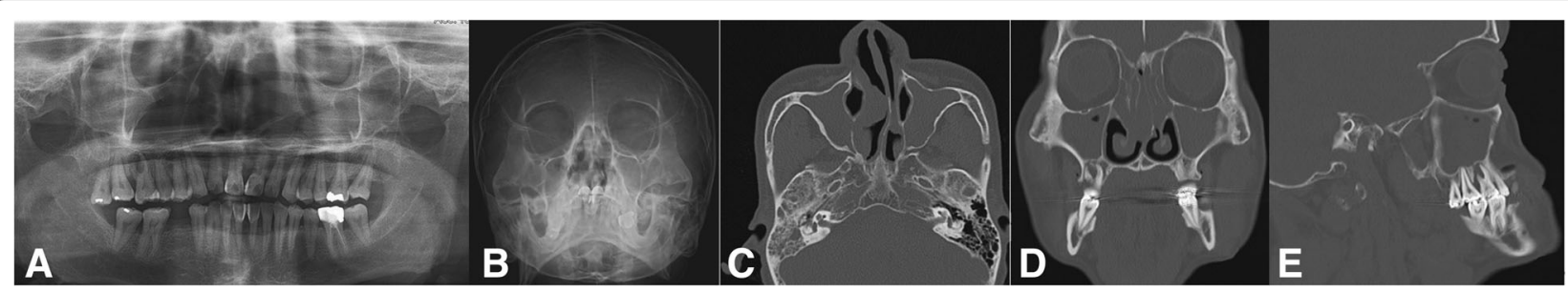

Fig. 9 Pan-sinusitis on both paranasal sinuses originating from the root infection of the right first molar. Preoperative panoramic view (a), Water's view (b), axial $\subset$ scan view showing sinusitis of both maxillary sinuses $(\mathbf{c})$, coronal $C T$ scan view showing whole sinusitis including both ethmoidal and frontal sinuses (d), and sagittal CT scan view showing involvement of the root pathologic lesion of the right first molar (e)

Main treatment with surgical approaches should be considered for the radical removal of any predisposing causes and for the restoration of normal MCC function. As most of these patients will be clinically immunodeficient or hospitalized, more delicate attention is required for the identification of early signs or symptoms.

\section{Management of odontogenic maxillary sinusitis Early diagnosis with management}

Although chronic sinusitis, including CRS and OMS, is common, accurate and early diagnosis is essential for successful management. In general, CRS will not initially cause facial pain, and a dentist may miss CRS in orofacial pain patients. Initial treatment such as nasal irrigation combined with the application of nasal decongestants should proceed after establishing the presence of nasal obstructions or polyps under endoscopic examination or via CT scans. If polyps are present, topical or systemic steroids should first be prescribed, and very limited use of nasal decongestants might be recommended. For surgical considerations of CSD or recurrent sinus disease, restoration of normal MCC function and clear opening of the OMU should be demonstrated first [56]. Several abnormal conditions, such as deviated septum, blocked polyp or turbinate, increased size of ostium, and hypertrophic middle meatus tissues should be managed by an otorhinolaryngologist using endoscopic views. After this initial management, odontogenic causes should be explored by the dentist or maxillofacial surgeon.

Mucous retention cysts are frequently found on panoramic views and CT scans of the floor of the maxillary sinus and are often confused with odontogenic inflammatory cysts (Figs. 5 and 6). Despite the fact that often, no treatment is recommended, retention cysts can be easily removed with an endoscopic-assisted approach due to their enlarging and non-self-remission characteristics. Mucoceles are found frequently in the maxillary sinus and are mostly located in the frontal sinus when sinus drainage is blocked. They occur when secreted mucus collects and leads to bony expansion with a strong pressure effect [57]. CRS that occurs after receiving high-dose radiation or in patients with cystic fibrosis may also require early management due to thick mucinous secretions with recurrent scar formation $[5,6]$.

\section{Prompt management}

Unilateral continuous or recalcitrant discomfort with or without foul odor is common in OMS, but the comprehensive diagnosis of OMS by dentists is difficult. Excluding CT scans or cone-beam CT, panoramic and Water's view can be used for the identification of sinusitis of dental origin, and thus dental management alone may be adequate to resolve OMS at first, followed by subsequent surgical approaches including FESS or CLP. Facial pain with tension headache and temporomandibular joint disorder after upper respiratory tract infection may sometimes be mistaken for OMS, but the origin of pain from the sinus or nasal cavity should be assessed for accurate discrimination [58].

There are several classic surgical strategies for approaching the maxillary sinus, such as the CLP and ESS, and these methods continue to be chosen by many surgeons, although they are accompanied by many complications. CLP, confusingly termed the Caldwell Luc operation, is widely used due to easy access and quick relief of symptoms. However, two typical complications, such as the formation of postoperative maxillary cysts (POMC) and high rates of inferior osteotomy blockade, are often inevitable. In addition, high incidences of postoperative facial swelling due to intraoperative hemorrhage, facial or teeth paresthesia from infraorbital nerve involvement, and sclerosis of the maxillary sinus wall occur after classic CLP [59-61] (Fig. 10a). Furthermore, such treatment of unexpected situations makes it difficult to reconstruct the alveolar ridge for implant or prosthetic rehabilitation.

FESS has been recommended by rhinologists due to its several advantages, including wide and flexible approaches to the paranasal sinuses (PNS) without limitation to the maxillary sinus. Anatomical widening of the middle nasal meatus with whole removal of diseased tissues and pathogens could lead to the recovery of sinus function with low morbidity and preserve the inner sinus mucosa and remaining SM (Fig. 10b). FESS has gradually replaced CLP during the past several years, but is associated with 


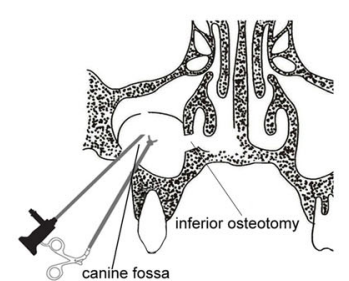

A

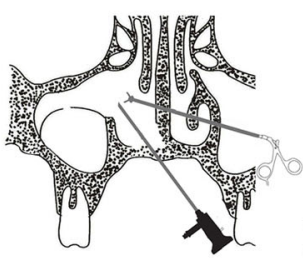

B

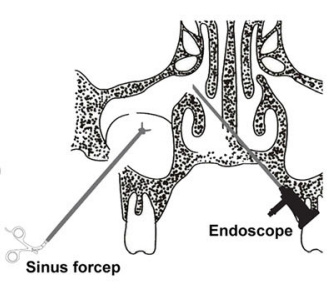

C

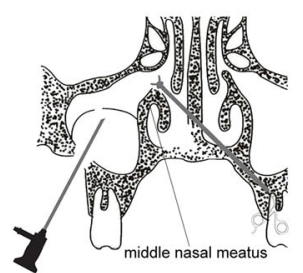

D

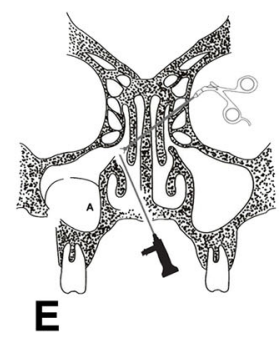

Fig. 10 Schematic drawings of surgical approaches in odontogenic maxillary sinusitis patients showing conventional CLP (a), FESS (b), and MESS (c, d, e)

complications [62]. However, the excess removal of inner physiologic tissues of the nasal cavity and incomplete solutions to odontogenic problems are deficiencies related to surgical options for OMS managements.

\section{Consolidated management}

Despite the development of FESS for CRS, consolidated management of OMS should ensure that the patient is infection-free without any recurrences. Complete removal of odontogenic origins, such as involved tooth extraction or apicoectomy with endodontic treatment, is essential for the prevention of OMS complications [15, 63, 64]. Additionally, due to the high frequency of OMS in the elderly, further considerations for bony reconstruction in the posterior maxillary alveolar ridge beneath the maxillary sinus are required for future prosthetic rehabilitation $[15,16]$.

Three main approaches can be used for the consolidated management of OMS: an intraoral approach through the originating tooth site, endoscopic approach through the nose and OMU, and intended upper maxilla approach after making bony window. Recently, modified endoscopy-assisted maxillary sinus surgery (MESS) was applied for the intraoral reduction of blowout orbital fractures [65], removal of sinus pathologies [66], and removal of migrated implants beneath the optic canal [67]. MESS is a new, innovative sinus-approach surgical procedure, which is efficient, easy, and less complicated than other sinus approaches (Fig. 10) Due to its capability to maintain sinus physiology and preserve the middle nasal meatus without causing POMC or sinus scar after CLP, OMU enlargement could be used for PNS ventilation into the nasal cavity.

\section{Prevention of anticipated complications}

OAF is the most common complication related to OMS. The main cause of OAF is the extraction of a maxillary posterior tooth, which accounts for more than $80 \%$ of all OAF cases [68-70]. This form of OAF is also referred to as oronasal fistula or oroantral communication, in which CRS may consequently occur via oral mucosal penetration between the posterior maxillary alveolus and the infero-lateral wall of the maxillary sinus. The main symptom of chronic non-healing OAF is purulent discharge through the fistula, especially when the patient drinks or blows through the nose from the OAF into the oral cavity or vice versa.

Regarding closure of the OAF, considerations of fistula size and depth are important for successful management. OAF can be self-covered with oral epithelium and granulation tissue or polyposis of the sinus mucosal membrane, but in cases of unsuccessful self-closure, hyperplasia of the sinus mucosal membrane can cause the formation of a very severe permanent fistula canal between the oral cavity and nose. Excluding the avoidance of OAF formation, the first solution for OAF would be CLP or FESS. The primary closure of OAF is determined according to defect size and health of the oral mucosa. Direct closure or extended surgical flaps, including buccal advancement, palatal island or pedicled flaps, may be considered for OAF management (Fig. 11). Additionally, the use of an absorbable barrier membrane, gold foils, or buccal fat pad closure could also be considered for severe OAF cases [71]. In every OAF case, maintaining a disease-free maxillary sinus membrane without infective microorganisms is also important for the functional recovery of the maxillary sinus [70-72].

\section{Conclusions}

The incidence of OMS is much higher than previously reported and occurs in more than $30-40 \%$ of all CMS cases. Although the exact etiopathogenesis of OMS is still uncertain, common causes are known to be iatrogenic and related to dental treatment of a posterior maxillary tooth or implant procedures. An infected SM with communication to the originating dental elements may demonstrate $\mathrm{BB}$ formation and should be checked first. Early endoscopic and radiographic investigations by otorhinolaryngologists should be followed by dentists with intraoral diagnoses made using panoramic or Water's views in chronic recalcitrant CRS patients. When considering treatment options for OMS, innovative approaches should be considered over conventional CLP, FESS, and MESS, due to lower rates of complications and better antral lining preservation. 

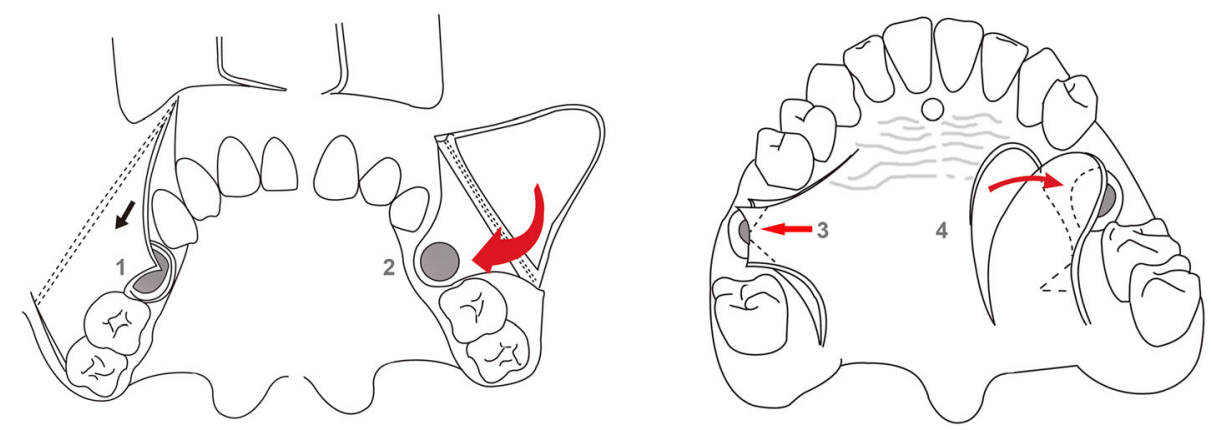

Fig. 11 Schematic drawings of an oroantral fistula closure in the oral cavity. Direct closure (1), buccal flap (2), palatal releasing flap (3), and palatal rotational pedicled flap (4)

\section{Abbreviations}

ABRS: Acute bacterial rhinosinusitis; ARS: Acute rhinosinusitis; BB: Bacterial biofilm; CLP: Caldwell Luc procedure; CMS: Chronic maxillary sinusitis; CRS: Chronic rhinosinusitis; FESS: Functional endoscopic sinus surgery; $H$. influenza: Haemophilus influenza; MCC: Mucociliary clearance; MESS: Modified endoscopy-assisted maxillary sinus surgen; OAF: Oroantral fistula; OMS: Odontogenic maxillary sinusitis; OMU: Osteo-meatal unit; P. aeruginosa: Pseudomonas aeruginosa; PNS: Paranasal sinuses; POMC: Postoperative maxillary cysts; PRISMA: Preferred Reporting Items for Systematic reviews and Meta-Analyses; S. aureus: Staphylococcus aureus; S. pneumonia: Streptococcus pneumonia; SM: Schneiderian membrane; SRS: Subacute rhinosinusitis; URI: Upper respiratory tract infection

\section{Acknowledgements}

This study was supported by grant No 01-2017-0010 from the SNUDH Research Fund and by Basic Science Research Program through the National Research Foundation of Korea funded by the Ministry of Education (2017R1D1A1B04029339).

\section{Funding}

There is no funding related to this article.

\section{Availability of data and materials}

Data sharing is not applicable to this article as no data sets were generated or analysed during the current study.

\section{Author's contributions}

SM read and wrote the whole manuscript. The author read and approved the final manuscript.

\section{Ethics approval and consent to participate}

Not applicable. But, for the clinical radiographic pictures included, we have received a statement of ethics approval was provided by the Department of Oral and Maxillofacial Surgery at Seoul National University Dental Hospital, with the approval of the Institutional Review Board of Seoul National University (S-D20170005).

\section{Consent for publication}

Written informed consent was obtained from the patients for the publication.

\section{Competing interests}

The author declares that there are no competing interests.

\section{Publisher's Note}

Springer Nature remains neutral with regard to jurisdictional claims in published maps and institutional affiliations.
Received: 6 January 2019 Accepted: 22 February 2019

Published online: 29 March 2019

\section{References}

1. Gaudin RA, Hoehle LP, Smeets R, Heiland M, Caradonna DS, Gray ST, Sedaghat AR (2018) Impact of odontogenic chronic rhinosinusitis on general health-related quality of life. Eur Arch Otorhinolaryngol 275: 1477-1482

2. Sarber KM, Dion GR, Weitzel EK, McMains KC (2013) Approaching chronic sinusitis. South Med J 106:642-648

3. Holt GR (2013) Rhinitis, acute sinusitis, and chronic sinusitis affect quality of life. South Med J 106:491

4. Dion GR, Weitzel EK, McMains KC (2013) Current approaches to diagnosis and management of rhinitis. South Med J 106:526-531

5. Stevens WW, Lee RJ, Schleimer RP, Cohen NA (2015) Chronic rhinosinusitis pathogenesis. J Allergy Clin Immunol 136:1442-1453

6. Dykewicz MS, Hamilos DL (2010) Rhinitis and sinusitis. J Allergy Clin Immunol 125:S103-S115

7. Dass K, Peters AT (2016) Diagnosis and management of rhinosinusitis: highlights from the 2015 practice parameter. Curr Allergy Asthma Rep 16:29

8. Hamilos DL (2011) Chronic rhinosinusitis: epidemiology and medical management. J Allergy Clin Immunol 128:693-707

9. Lopatin AS, Sysolyatin SP, Sysolyatin PG, Melnikov MN (2002) Chronic maxillary sinusitis of dental origin: is external surgical approach mandatory? Laryngoscope 112:1056-1059

10. An JH, Park SH, Han JJ, Jung S, Kook MS, Park HJ, Oh HK (2017) Treatment of dental implant displacement into the maxillary sinus. Maxillofac Plast Reconstr Surg 25 39:35

11. Melen I, Lindahl L, Andréasson L, Rundcrantz H (1986) Chronic maxillary sinusitis. Definition, diagnosis and relation to dental infections and nasal polyposis. Acta Otolaryngol 101:320-327

12. Albu S, Baciut M (2010) Failures in endoscopic surgery of the maxillary sinus. Otolaryngol Head Neck Surg 142:196-201

13. Kretzschmar DP, Kretzschmar J (2003) Rhinosinusitis: review from a dental perspective. Oral Surg Oral Med Oral Pathol Oral Radiol Endod 96:128-135

14. Bauer WH (1943) Maxillary sinusitis of dental origin. Am J Orthod Oral Surg 29:133-151

15. Brook I (2006) Sinusitis of odontogenic origin. Otolaryngol Head Neck Surg 135:349-355

16. Mehra P, Jeong D (2008) Maxillary sinusitis of odontogenic origin. Curr Infect Dis Rep 10:205-210

17. Simuntis R, Kubilius $R$, Padervinskis E, Ryškienè $S$, Tušas $P$, Vaitkus $S$ (2017) Clinical efficacy of main radiological diagnostic methods for odontogenic maxillary sinusitis. Eur Arch Otorhinolaryngol 274:3651-3658

18. Oberli K, Bornstein MM, von Arx T (2007) Periapical surgery and the maxillary sinus: radiographic parameters for clinical outcome. Oral Surg Oral Med Oral Pathol Oral Radiol Endod 103:848-853

19. Göçmen G, Varol A, Göker K, Basa S (2011) Actinomycosis: report of a case with a persistent extraoral sinus tract. Oral Surg Oral Med Oral Pathol Oral Radiol Endod 112:e121-e123 
20. Arias-Irimia O, Barona-Dorado C, Santos-Marino JA, Martínez-Rodriguez N, Martínez-González JM (2010) Metaanalysis of the etiology of odontogenic maxillary sinusitis. Med Oral Patol Oral Cir Bucal 5:e70-e73

21. Kim SB, Yun PY, Kim YK (2016) Clinical evaluation of sinus bone graft in patients with mucous retention cyst. Maxillofac Plast Reconstr Surg 25 38:35

22. Longhini AB, Ferguson BJ (2011) Clinical aspects of odontogenic maxillary sinusitis: a case series. Int Forum Allergy Rhinol 1:409-415

23. Legert KG, Zimmerman M, Stierna P (2004) Sinusistis of odonyogenic origin: pathophysiological implications of early treatment. Acta Otolaryngol 124:655-663

24. Figdor D, Gulabivala K (2011) Survival against the odds: microbiology of root canals associated with post-treatment disease. Endod Topics 18:62-77

25. Ricucci D, Siqueira JF Jr (2008) Apical actinomycosis as a continuum of intraradicular and extraradicular infection: case report and critical review on its involvement with treatment failure. J Endod 34:1124-1129

26. Su L, Gao Y, Yu C, Wang H, Yu Q (2010) Surgical endodontic treatment of refractory periapical periodontitis with extraradicular biofilm. Oral Surg Ora Med Oral Pathol Oral Radiol Endod 110:e40-e44

27. Selden HS (1974) The interrelationship between the maxillary sinus and endodontics. Oral Surg Oral Med Oral Pathol 4:623-627

28. Selden HS (1989) The endo-antral syndrome: an endodontic complication. J Am Dent Assoc 119:397-402

29. Selden HS (1999) Endo-antral syndrome and various endodontic complications. J Endod 25:389-393

30. Dugas NN, Lawrence HP, Teplitsky PE, Pharoah MJ, Friedman (2003) Periapical health and treatment quality assessment of root-filled teeth in two Canadian populations. Int Endod J 36:181-192

31. Nair PNR (2006) On the causes of persistent apical periodontitis: a review. Int Endod J 39:249-281

32. Nair PNR, Pajarola G, Schroeder HE (1996) Types and incidence of human periapical lesions obtained with extracted teeth. Oral Surg Oral Med Oral Pathol Oral Radiol Endod 81:93-102

33. Yanagisawa W (1980) Pathologic study of periapical lesions. I. Periapical granulomas: clinical, histologic and immunohistopathologic studies. J Oral Pathol 9:288-300

34. Nair PNR, Schmid-Meier E (1986) An apical granuloma with epithelial integument. Oral Surg Oral Med Oral Pathol Oral Radiol Endod 62:698-703

35. Haapasalo M, Shen Y, Ricucci D (2011) Reasons for persistent and emerging post-treatment endodontic disease. Endod Topics 18:31-50

36. Matilla K (1965) Roentgenological investigations of the relationship between periapical lesions and conditions of the mucous membrane of the maxillary sinuses. Acta Odontol Scand 23:42-46

37. Matilla K, Altonen MA (1968) A clinical and roentgenological study of apicectomized teeth. Odontol Tidskr 76:389-406

38. Aruni AW, Dou Y, Mishra A, Fletcher HM (2015) The biofilm communityrebels with a cause. Curr Oral Health Rep 2:48-56

39. Post C, Stoodley P, Hall-Stoodley L, Erlich G (2004) The role of biofilms in otolaryngologic infections. Curr Opin Otolaryngol Head Neck Surg 12:185-190

40. Foreman A, Psaltis AJ, Tan LW, Wormald PJ (2009) Characterization of bacterial and fungal biofilms in chronic rhinosinusitis. Am J Rhinol Allergy 23:556-561

41. Healy DY, Leid JG, Sanderson AR, Hunsaker DH (2008) Biofilms with fungi in chronic rhinosinusitis. Otolaryngol Head Neck Surg 138:641-647

42. Sunde PT, Olsen I, Debelian GJ, Tronstad L (2002) Microbiota of periapical lesions refractory to endodontic therapy. J Endod 28:304-310

43. Ramadan HH, Sanclement JA, Thomas JG (2005) Chronic rhinosinusitis and biofilms. Otolaryngol Head Neck Surg 132:414-417

44. Sanclement JA, Webster P, Thomas J, Ramadan HH (2005) Bacterial biofilms in surgical specimens of patients with chronic rhinosinusitis. Laryngoscope 115:578-582

45. Chen HH, Liu X, Ni C, Lu YP, Xiong GY, Lu YY, Wang SQ (2012) Bacterial biofilms in chronic rhinosinusitis and their relationship with inflammation severity. Auris Nasus Larynx 39:169-174

46. Prince AA, Steiger JD, Khalid AN, Dogrhamji L, Reger C, Eau Claire S, Chiu AG, Kennedy DW, Palmer JN, Cohen NA (2008) Prevalence of biofilmforming bacteria in chronic rhinosinusitis. Am J Rhinol 22:239-245

47. Tatar EC, Tatar I, Ocal B, Korkmaz H, Saylam G, Ozdek A, Celik HH (2012) Prevalence of biofilms and their response to medical treatment in chronic rhinosinusitis without polyps. Otolaryngol Head Neck Surg 146:669-675
48. Ragab A, Essa N, El-Raghy N, Zahran W, El Borolsy A (2012) Evaluation of bacterial adherence and biofilm arrangements as new targets in treatment of chronic rhinosinusitis. Eur Arch Otorhinolaryngol 269:537-544

49. Mladina R, Skitareli N, Musi S, Risti M (2010) A biofilm exists on healthy mucosa of the paranasal sinuses: a prospectively performed, blinded, scanning electron microscope study. Clin Otolaryngol 35:104-110

50. Perloff JR, Palmer JN (2004) Evidence of bacterial biofilms on frontal recess stents in patients with chronic rhinosinusitis. Am J Rhinol 18:377-380

51. Hunsaker DH, Leid JG (2008) The relationship of biofilms to chronic rhinosinusitis. Curr Opin Otolaryngol Head Neck Surg 16:237-241

52. Whittaker CJ, Klier CM, Kolenbrander PE (1996) Mechanisms of adhesion by oral bacteria. Annu Rev Microbiol 50:513-552

53. Hallberg K, Holm C, Ohman U, Stromberg N (1998) Actinomyces naeslundii displays variant fimP and fimA fimbrial subunit genes corresponding to different types of acidic proline-rich protein and beta-linked galactosamine binding specificity. Infect Immun 66:4403-4410

54. Sunde PT, Tronstad L, Eribe ER, Lind PO, Olsen I (2000) Assessment of periradicular microbiota by DNA-DNA hybridization. Endod Dent Traumatol 16:191-196

55. Grosjean P, Weber R (2007) Fungus balls of the paranasal sinuses: a review. Eur Arch Otorhinolaryngol 264:461-470

56. Manarey CR, Anand VK, Huang C (2004) Incidence of methicillinresistant Staphylococcus aureus causing chronic rhinosinusitis. Laryngoscope 114:939-941

57. Kang IG, Kim ST, Jung JH, Paik JY, Woo JH, Cha HE, Chi MJ, Jin SM, Lee KC (2014) Effect of endoscopic marsupialization of paranasal sinus mucoceles involving the orbit: a review of 27 cases. Eur Arch Otorhinolaryngol 271:293-297

58. Bell GW, Joshi BB, Macleod RI (2011) Maxillary sinus disease: diagnosis and treatment. Br Dent J 210:113-118

59. Vassallo P, Tranfa F, Forte R, D'Aponte A, Strianese D, Bonavolontà G (2001) Ophthalmic complications after surgery for nasal and sinus polyposis. Eur J Ophthalmol 11:218-222

60. DeFreitas J, Lucente FE (1998) The Caldwell-Luc procedure: institutional review of 670 cases: 1975-1985. Laryngoscope 98:1297-1300

61. Low WK (1995) Complications of the Caldwell-Luc operation and how to avoid them. Aust N Z J Surg 6:582-584

62. Närkiö-Mäkelä M, Qvarnberg Y (1997) Endoscopic sinus surgery or CaldwellLuc operation in the treatment of chronic and recurrent maxillary sinusitis. Acta Otolaryngol Suppl 529:177-180

63. Konstantinidis I, Constantinidis J (2014) Medial maxillectomy in recalcitrant sinusitis: when, why and how? Curr Opin Otolaryngol Head Neck Surg 22:68-74

64. Simuntis R, Kubilius R, Vaitkus S (2014) Odontogenic maxillary sinusitis: a review. Stomatologija 16:39-43

65. Park MW, Kim SM, Amponsah EK, Lee SK (2015) Simple repair of a blow-out fracture by the modified Caldwell-Luc approach. J Craniofac Surg 26:e306-e330

66. Kim SM, Eo MY, Cho YJ, Kim YS, Lee SK (2017) Differential protein expression in the secretory fluids of maxillary sinusitis and maxillary retention cyst. Eur Arch Otorhinolaryngol 274:215-222

67. Kim SM (2017) The removal of an implant beneath the optic canal by modified endoscopic-assisted sinus surgery. Eur Arch Otorhinolaryngol 274: $1167-1171$

68. Sokler K, Vuksan V, Lauc T (2002) Treatment of oroantral fistula. Acta Stomat Croat 36:135-140

69. Fusetti S, Emanuelli E, Ghirotto C, Bettini G, Ferronato G (2013) Chronic oroantral fistula: combined endoscopic and intraoral approach under local anesthesia. Am J Otolaryngol 34:323-326

70. Kim MK, Han W, Kim SG (2017) The use of the buccal fat pad flap for oral reconstruction. Maxillofac Plast Reconstr Surg 25 39:5

71. Horowitz G, Koren I, Carmel NN, Balaban S, Abu-Ghanem S, Fliss DM, Kleinman S, Reiser V (2016) One stage combined endoscopic and per-oral buccal fat pad approach for large oro-antral-fistula closure with secondary chronic maxillary sinusitis. Eur Arch Otorhinolaryngol 273:905-909

72. Hajiioannou J, Koudounarakis E, Alexopoulos K, Kotsani A, Kyrmizakis DE (2010) Maxillary sinusitis of dental origin due to oroantral fistula, treated by endoscopic sinus surgery and primary fistula closure. J Laryngol Otol 124: 986-989 